RIMS Preprint No. 1470, Kyoto University, August 2004

\title{
A Two-Sided Discrete-Concave Market with Bounded Side Payments: An Approach by Discrete Convex Analysis
}

\author{
Satoru Fujishige and Akihisa Tamura
}

\begin{abstract}
The marriage model due to Gale and Shapley and the assignment model due to Shapley and Shubik are standard in the theory of two-sided matching markets. We give a common generalization of these models by utilizing discrete concave functions and considering bounded side payments. We show the existence of a pairwise stable outcome in our model. Our present model is a further natural extension of the model examined in our previous paper (Fujishige and Tamura [10]), and the proof of the existence of a pairwise stable outcome is even simpler than the previous one.
\end{abstract}

Satoru Fujishige

Research Institute for Mathematical Sciences, Kyoto University, Kyoto 606-8502, Japan.

phone: +81-75-753-7250, facsimile: +81-75-753-7272

e-mail: fujishig@kurims.kyoto-u.ac.jp.

Akihisa Tamura

Research Institute for Mathematical Sciences,

Kyoto University, Kyoto 606-8502, Japan.

phone: +81-75-753-7236, facsimile: +81-75-753-7272

e-mail: tamura@kurims.kyoto-u.ac.jp. 


\section{Introduction}

The marriage model due to Gale and Shapley [12] and the assignment model due to Shapley and Shubik [26] are standard in the theory of two-sided matching markets. The largest difference between these two models is that the former does not allow money or transferable utilities whereas the latter does (see Roth and Sotomayor [24], Alkan and Gale [1] and Fleiner [8] for related models).

Progress has been made toward unifying the marriage model and the assignment model. Kaneko [15] formulated a general model that includes the two by means of characteristic functions, and proved the nonemptiness of the core. Roth and Sotomayor [25] proposed a general model that also encompasses both and investigated the lattice property for payoffs. Eriksson and Karlander [4] proposed a hybrid model of the marriage model and the assignment model. In the ErikssonKarlander model, the set of agents is partitioned into two categories, one for "rigid" agents and the other for "flexible" agents. Rigid agents do not get side payments, that is, they behave like agents in the marriage model, while flexible agents behave like ones in the assignment model. Sotomayor [27] also further investigated this hybrid model and gave a non-constructive proof of the existence of a pairwise stable outcome. Fujishige and Tamura [10] proposed a generalization of the hybrid model due to Eriksson and Karlander [4] and Sotomayor [27] by utilizing discrete convex analysis developed by Murota [18, 19, 20].

Our goal is to propose a further general model based on discrete convex analysis which includes models due to Gale and Shapley [12], Shapley and Shubik [26], Eriksson and Karlander [4], Sotomayor [27], Fleiner [7], Eguchi et al. [3], and Fujishige and Tamura [10] as special cases, and to verify the existence of a pairwise stable outcome in our model. The feature of our model is to adopt a range of a side payment for each pair of agents instead of using the concept of "rigid" and "flexible" agents. Our present model is a natural extension of the model of Fujishige and Tamura [10], and our proof for the existence of a pairwise stable outcome is simpler than that in our previous paper.

The present paper is organized as follows. Section 2 explains $\mathrm{M}^{\natural}$-concavity together with some examples. Section 3 describes our model based on discrete convex analysis and gives our main theorem. In Section 4 we give proofs of two lemmas mentioned in Section 3, and propose an algorithm for finding a pairwise stable outcome in our model. By proving its correctness, we show our main theorem, the existence of a pairwise stable outcome. 


\section{2. $M^{\natural}$-concavity}

In this section we explain the concept of $\mathrm{M}^{\natural}$-concave functions. Let $E$ be a nonempty finite set, and let $\mathbf{Z}$ and $\mathbf{R}$ be the sets of integers and reals, respectively. We denote by $\mathbf{Z}^{E}$ the set of integer vectors $x=(x(e) \mid e \in E)$ indexed by $E$, where $x(e)$ denotes the eth component of vector $x$. Also, $\mathbf{R}^{E}$ denotes the set of real vectors indexed by $E$. Let $\mathbf{0}$ and $\mathbf{1}$ be vectors of all zeros and all ones of an appropriate dimension. We define the positive support $\operatorname{supp}^{+}(x)$ and the negative

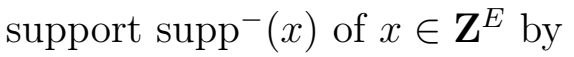

$$
\operatorname{supp}^{+}(x)=\{e \in E \mid x(e)>0\}, \operatorname{supp}^{-}(x)=\{e \in E \mid x(e)<0\} .
$$

For each $S \subseteq E$, we denote by $\chi_{S}$ the characteristic vector of $S$ defined by: $\chi_{S}(e)=1$ if $e \in S$ and $\chi_{S}(e)=0$ otherwise, and write simply $\chi_{e}$ instead of $\chi_{\{e\}}$ for all $e \in E$. For $S \subseteq E$ and $x \in \mathbf{Z}^{E}$, let $x(S)=\sum_{e \in S} x(e)$. For a vector $p \in \mathbf{R}^{E}$ and a function $f: \mathbf{Z}^{E} \rightarrow \mathbf{R} \cup\{-\infty\}$, we define functions $\langle p, x\rangle$ and $f[p](x)$ in $x \in \mathbf{Z}^{E}$ by

$$
\langle p, x\rangle=\sum_{e \in E} p(e) x(e), \quad f[p](x)=f(x)+\langle p, x\rangle \quad\left(\forall x \in \mathbf{Z}^{E}\right) .
$$

We also define $\arg \max$, the set of maximizers, of $f$ on $U \subseteq \mathbf{Z}^{E}$ and the effective domain of $f$ by

$$
\begin{aligned}
& \arg \max \{f(y) \mid y \in U\}=\{x \in U \mid \forall y \in U: f(x) \geq f(y)\}, \\
& \operatorname{dom} f=\left\{x \in \mathbf{Z}^{E} \mid f(x)>-\infty\right\}
\end{aligned}
$$

A function $f: \mathbf{Z}^{E} \rightarrow \mathbf{R} \cup\{-\infty\}$ with $\operatorname{dom} f \neq \emptyset$ is called $M^{\natural}$-concave (Murota [20] and Murota and Shioura [21]) if it satisfies

$\left(\mathrm{M}^{\natural}\right) \forall x, y \in \operatorname{dom} f, \forall e \in \operatorname{supp}^{+}(x-y), \exists e^{\prime} \in \operatorname{supp}^{-}(x-y) \cup\{0\}:$

$$
f(x)+f(y) \leq f\left(x-\chi_{e}+\chi_{e^{\prime}}\right)+f\left(y+\chi_{e}-\chi_{e^{\prime}}\right),
$$

where 0 is a new element not in $E$ and $\chi_{0}$ is a zero vector in $\mathbf{Z}^{E}$.

$\left(\left(\mathrm{M}^{\natural}\right)\right.$ is denoted by $\left(-\mathrm{M}^{\natural}-\mathrm{EXC}\right)$ in Murota [20].) If $f$ is $\mathrm{M}^{\natural}$-concave, then $f[p]$ is also $\mathrm{M}^{\natural}$-concave for any $p \in \mathbf{R}^{E}$. Here are two simple examples of $\mathbf{M}^{\natural}$-concave functions.

Example 1: For the independence family $\mathcal{I} \subseteq 2^{E}$ of a matroid on $E$ and $w \in \mathbf{R}^{E}$, the function $f: \mathbf{Z}^{E} \rightarrow \mathbf{R} \cup\{-\infty\}$ defined by

$$
f(x)=\left\{\begin{array}{ll}
\sum_{e \in X} w(e) & \text { if } x=\chi_{X} \text { for some } X \in \mathcal{I} \\
-\infty & \text { otherwise }
\end{array} \quad\left(\forall x \in \mathbf{Z}^{E}\right)\right.
$$

is $\mathrm{M}^{\natural}$-concave (see Murota [20]). 
Example 2: We call a nonempty family $\mathcal{T}$ of subsets of $E$ a laminar family if $X \cap Y=\emptyset, X \subseteq Y$ or $Y \subseteq X$ holds for any $X, Y \in \mathcal{T}$. For a laminar family $\mathcal{T}$ and a family of univariate concave functions $f_{Y}: \mathbf{R} \rightarrow \mathbf{R} \cup\{-\infty\}$ indexed by $Y \in \mathcal{T}$, the function $f: \mathbf{Z}^{E} \rightarrow \mathbf{R} \cup\{-\infty\}$ defined by

$$
f(x)=\sum_{Y \in \mathcal{T}} f_{Y}(x(Y)) \quad\left(\forall x \in \mathbf{Z}^{E}\right)
$$

is $\mathrm{M}^{\natural}$-concave if $\operatorname{dom} f \neq \emptyset$ (see Murota [20]).

An $\mathrm{M}^{\natural}$-concave function has nice features as a utility function from the point of view of mathematical economics. A utility function is usually assumed to be concave in that field. For any $\mathrm{M}^{\natural}$-concave function $f: \mathbf{Z}^{E} \rightarrow \mathbf{R} \cup\{-\infty\}$, there exists an ordinary concave function $\bar{f}: \mathbf{R}^{E} \rightarrow \mathbf{R} \cup\{-\infty\}$ such that $\bar{f}(x)=f(x)$ for all $x \in \mathbf{Z}^{E}$ (Murota [18]). That is, any $\mathrm{M}^{\natural}$-concave function on $\mathbf{Z}^{E}$ has a concave extension on $\mathbf{R}^{E}$. An $\mathrm{M}^{\natural}$-concave function $f$ also satisfies submodularity (Murota and Shioura [22]): $f(x)+f(y) \geq f(x \wedge y)+f(x \vee y)$ for all $x, y \in \operatorname{dom} f$, where $x \wedge y$ and $x \vee y$ are the vectors whose eth components $(x \wedge y)(e)$ and $(x \vee y)(e)$ are, respectively, $\min \{x(e), y(e)\}$ and $\max \{x(e), y(e)\}$ for all $e \in E$. Moreover, an $\mathrm{M}^{\natural}$-concave function satisfies gross substitutability and the single improvement property discussed in Kelso and Crawford [16] and Gul and Stacchetti [13], and $\mathrm{M}^{\natural}$-concavity can be characterized by these properties or their extensions under a natural assumption (see Fujishige and Yang [11], Danilov et al. [2] and Murota and Tamura [23] for details). Relations between $\mathrm{M}^{\natural}$-concavity and substitutability are also discussed in Farooq and Tamura [6] and Farooq and Shioura [5].

\section{Model description}

We consider a two-sided market consisting of disjoint sets $P$ and $Q$ of agents, in which an agent in $P$ may be called a worker and one in $Q$ a firm. Each worker $i \in P$ can supply multi-units of labor time, and each firm $j \in Q$ can employ workers with multi-units of labor time and pay a salary to worker $i$ per unit of labor time if $j$ hires $i$. We assume bounded side payments, i.e., each pair $(i, j)$ has lower and upper bounds on a salary per unit of labor time. We also assume that utility of each worker $i \in P$ and profit of each firm $j \in Q$ are described by functions in monetary terms. We will examine pairwise stability in this market.

We first describe our model mathematically. Let $E=P \times Q$, i.e., the set of all ordered pairs $(i, j)$ of agents $i \in P$ and $j \in Q$. Also define $E_{(i)}=\{i\} \times Q$ for all $i \in P$ and $E_{(j)}=P \times\{j\}$ for all $j \in Q$. Denoting by $x(i, j)$ the number of units of labor time for which $j$ hires $i$, we represent a labor allocation by vector $x=(x(i, j) \mid(i, j) \in E) \in \mathbf{Z}^{E}$. We express lower and upper bounds of salaries 
by two vectors $\underline{\pi} \in(\mathbf{R} \cup\{-\infty\})^{E}$ and $\bar{\pi} \in(\mathbf{R} \cup\{+\infty\})^{E}$ with $\underline{\pi} \leq \bar{\pi}$. For any $y \in \mathbf{R}^{E}$ and $k \in P \cup Q$, we denote by $y_{(k)}$ the restriction of $y$ on $E_{(k)}$. For example, for a labor allocation $x \in \mathbf{Z}^{E}, x_{(k)}$ represents the labor allocation of agent $k$ with respect to $x$. For each $i \in P$ and $j \in Q$, let $f_{i}: \mathbf{Z}^{E_{(i)}} \rightarrow \mathbf{R} \cup\{-\infty\}$ denote utility of worker $i$ and $f_{j}: \mathbf{Z}^{E_{(j)}} \rightarrow \mathbf{R} \cup\{-\infty\}$ profit of firm $j$. We assume that for each $k \in P \cup Q$ function $f_{k}$ satisfies the following assumption:

(A) $\operatorname{dom} f_{k}$ is bounded and hereditary, and has $\mathbf{0}$ as the minimum point,

where heredity means that for any $y, y^{\prime} \in \mathbf{Z}^{E_{(k)}}, \mathbf{0} \leq y^{\prime} \leq y \in \operatorname{dom} f_{k}$ implies $y^{\prime} \in \operatorname{dom} f_{k}$. The boundedness of effective domains means that each function is implicitly imposed on firm's budget constraint or worker's constraint on labor time. The heredity of effective domains implies that each agent can arbitrarily decrease related labor time (before contract) without any permission from the partner.

A vector $x \in \mathbf{Z}^{E}$ is called a feasible allocation if $x_{(k)} \in \operatorname{dom} f_{k}$ for all $k \in P \cup Q$. Given a feasible allocation $x$, a vector $s \in \mathbf{R}^{E}$ is called an $x$-compatible salary vector if $\underline{\pi}(i, j) \leq s(i, j) \leq \bar{\pi}(i, j)$ for all $(i, j) \in E$ with $x(i, j)>0$ and if $s(i, j)=0$ for all $(i, j) \in E$ with $x(i, j)=0$. We call a pair $(x, s)$ of a feasible allocation $x \in \mathbf{Z}^{E}$ and an $x$-compatible salary vector $s \in \mathbf{R}^{E}$ an outcome.

An outcome $(x, s)$ is said to satisfy incentive constraints if

$$
\begin{aligned}
& f_{i}\left[+s_{(i)}\right]\left(x_{(i)}\right)=\max \left\{f_{i}\left[+s_{(i)}\right](y) \mid y \leq x_{(i)}\right\} \quad(\forall i \in P), \\
& f_{j}\left[-s_{(j)}\right]\left(x_{(j)}\right)=\max \left\{f_{j}\left[-s_{(j)}\right](y) \mid y \leq x_{(j)}\right\} \quad(\forall j \in Q) \text {. }
\end{aligned}
$$

Here $f_{i}\left[+s_{(i)}\right]\left(x_{(i)}\right)$ is the sum of $i$ 's monetary utility for labor allocation $x$ and the amount of $i$ 's salary for $(x, s)$, and hence, it means the net payoff of worker $i$ with respect to $(x, s)$. Similarly, $f_{j}\left[-s_{(j)}\right]\left(x_{(j)}\right)$ mean the net profit of firm $j$ with respect to $(x, s)$. Conditions (3.1) and (3.2) say that each agent has no incentive to decrease the current units of labor time for the current salaries.

For $s \in \mathbf{R}^{E}, \alpha \in \mathbf{R}, i \in P$, and $j \in Q$, let $\left(s_{(i)}^{-j}, \alpha\right)$ be defined as the vector obtained from $s_{(i)}$ by replacing its $j$ th component by $\alpha$, and $\left(s_{(j)}^{-i}, \alpha\right)$ be similarly defined. An outcome $(x, s)$ is called pairwise unstable if it does not satisfy incentive constraints or there exist $i \in P, j \in Q, \alpha \in[\underline{\pi}(i, j), \bar{\pi}(i, j)], y^{\prime} \in \mathbf{Z}^{E_{(i)}}$ and $y^{\prime \prime} \in \mathbf{Z}^{E_{(j)}}$ such that

$$
\begin{aligned}
& f_{i}\left[+s_{(i)}\right]\left(x_{(i)}\right)<f_{i}\left[+\left(s_{(i)}^{-j}, \alpha\right)\right]\left(y^{\prime}\right), \\
& y^{\prime}\left(i, j^{\prime}\right) \leq x\left(i, j^{\prime}\right) \quad\left(\forall j^{\prime} \in Q \backslash\{j\}\right), \\
& f_{j}\left[-s_{(j)}\right]\left(x_{(j)}\right)<f_{j}\left[-\left(s_{(j)}^{-i}, \alpha\right)\right]\left(y^{\prime \prime}\right), \\
& y^{\prime \prime}\left(i^{\prime}, j\right) \leq x\left(i^{\prime}, j\right) \quad\left(\forall i^{\prime} \in P \backslash\{i\}\right), \\
& y^{\prime}(i, j)=y^{\prime \prime}(i, j) .
\end{aligned}
$$


Conditions $(3.3) \sim(3.7)$ say that $i$ and $j$ can strictly increase their net payoff and profit by concertedly changing the current units of labor time and the current salary between them under the constraints that units of labor time of the other parts are not increased. An outcome $(x, s)$ is called pairwise stable if it is not pairwise unstable.

We also consider a stronger pairwise stability. We say that an outcome $(x, s)$ is pairwise quasi-unstable if it does not satisfy incentive constraints or there exist $i \in P, j \in Q, \alpha \in[\underline{\pi}(i, j), \bar{\pi}(i, j)], y^{\prime} \in \mathbf{Z}^{E_{(i)}}$ and $y^{\prime \prime} \in \mathbf{Z}^{E_{(j)}}$ satisfying (3.3) (3.6) (but not necessarily (3.7)). Trivially, a pairwise unstable outcome is pairwise quasiunstable. An outcome $(x, s)$ is called pairwise strictly stable if it is not pairwise quasi-unstable. Hence, an outcome $(x, s)$ is pairwise strictly stable if and only if (3.1) and (3.2) hold and for all $i \in P, j \in Q$ and $\alpha \in \mathbf{R}$ with $\underline{\pi}(i, j) \leq \alpha \leq \bar{\pi}(i, j)$,

$$
f_{i}\left[+s_{(i)}\right]\left(x_{(i)}\right) \geq \max \left\{f_{i}\left[+\left(s_{(i)}^{-j}, \alpha\right)\right](y) \mid y\left(i, j^{\prime}\right) \leq x\left(i, j^{\prime}\right), \forall j^{\prime} \neq j\right\}
$$

or

$$
f_{j}\left[-s_{(j)}\right]\left(x_{(j)}\right) \geq \max \left\{f_{j}\left[-\left(s_{(j)}^{-i}, \alpha\right)\right](y) \mid y\left(i^{\prime}, j\right) \leq x\left(i^{\prime}, j\right), \forall i^{\prime} \neq i\right\} .
$$

The above condition says that for each pair $(i, j) \in E$ both $i$ and $j$ do not have incentive to change the current feasible allocation for any candidate of a salary between them. Note that any pairwise strictly stable outcome is pairwise stable.

Example 3: There is a gap between pairwise stability and pairwise strict stability. Let us consider the case where $E=\{(m, w)\}$,

$$
\begin{aligned}
& f_{m}(x)=\left\{\begin{array}{cl}
x & \text { if } x \in\{0,1,2\} \\
-\infty & \text { otherwise }
\end{array} \quad(\forall x \in \mathbf{Z}),\right. \\
& f_{w}(x)=\left\{\begin{array}{cl}
x & \text { if } x \in\{0,1,2,3\} \\
-\infty & \text { otherwise }
\end{array} \quad(\forall x \in \mathbf{Z}),\right.
\end{aligned}
$$

and $(\underline{\pi}, \bar{\pi})=(0,1 / 4)$. In this case, an outcome $(x, s)=(2,0)$ is not pairwise strictly stable, because $f_{m}(2)<f_{m}[+\epsilon](2)$ and $f_{w}(2)<f_{w}[-\epsilon](3)$ for all $\epsilon \in$ $(0,1 / 4]$. However, the outcome is pairwise stable. On the other hand, an outcome $(x, s)=(2,1 / 4)$ is pairwise strictly stable, and hence, pairwise stable.

Pairwise strict stability coincides with pairwise stability under certain conditions.

Lemma 3.1: If $f_{k}(k \in P \cup Q)$ are $M^{\natural}$-concave functions satisfying (A) and if one of the following conditions

(i) $\underline{\pi}=\bar{\pi}$, 
(ii) $\operatorname{dom} f_{k} \subseteq\{0,1\}^{E_{(k)}}$ for all $k \in P \cup Q$,

(iii) dom $\sum_{i \in P} f_{i}=\operatorname{dom} \sum_{j \in Q} f_{j}=\left\{y \in \mathbf{Z}^{E} \mid \mathbf{0} \leq y \leq u\right\}$ for some vector $u$, and $f_{k}(k \in P \cup Q)$ are linear over $\operatorname{dom} f_{k}$

holds, then any pairwise stable outcome is pairwise strictly stable.

Proof. See Section 4.1.

The concept of pairwise stability in our model coincides, in special cases, with those in the marriage model, the assignment model, and so on (see Remarks 1 and 2 after Theorem 3.5).

The following theorem states that the existence of a pairwise strictly stable outcome, and hence, the existence of a pairwise stable outcome, is certified by $\mathrm{M}^{\natural}$-concave functions.

Theorem 3.2: For $M^{\natural}$-concave functions $f_{k}(k \in P \cup Q)$ satisfying (A) and for vectors $\underline{\pi} \in(\mathbf{R} \cup\{-\infty\})^{E}$ and $\bar{\pi} \in(\mathbf{R} \cup\{+\infty\})^{E}$ with $\underline{\pi} \leq \bar{\pi}$, there exists a pairwise strictly stable outcome $(x, s)$, and hence, there exists a pairwise stable outcome.

To show Theorem 3.2, we give an alternative characterization of a pairwise strictly stable outcome.

Lemma 3.3: Assume that $f_{k}$ is an $M^{\natural}$-concave function satisfying (A) for each $k \in P \cup Q$. Let $x$ be a feasible allocation. There exists an $x$-compatible salary vector $s$ forming a pairwise strictly stable outcome $(x, s)$ if and only if there exist $p \in \mathbf{R}^{E}$, $z_{P}=\left(z_{(i)} \mid i \in P\right) \in(\mathbf{Z} \cup\{+\infty\})^{E}$, and $z_{Q}=\left(z_{(j)} \mid j \in Q\right) \in(\mathbf{Z} \cup\{+\infty\})^{E}$ such that

$$
\begin{aligned}
& x_{(i)} \in \arg \max \left\{f_{i}\left[+p_{(i)}\right](y) \mid y \leq z_{(i)}\right\} \quad(\forall i \in P), \\
& x_{(j)} \in \arg \max \left\{f_{j}\left[-p_{(j)}\right](y) \mid y \leq z_{(j)}\right\} \quad(\forall j \in Q), \\
& \underline{\pi} \leq p \leq \bar{\pi}, \\
& e \in E, z_{P}(e)<+\infty \Rightarrow p(e)=\underline{\pi}(e), z_{Q}(e)=+\infty, \\
& e \in E, z_{Q}(e)<+\infty \Rightarrow p(e)=\bar{\pi}(e), z_{P}(e)=+\infty .
\end{aligned}
$$

Moreover, from any $p$ satisfying the above conditions, we can construct a required salary vector $s$ by defining $s(e)=p(e)$ for all $e \in E$ with $x(e)>0$ and $s(e)=0$ for all $e \in E$ with $x(e)=0$.

Proof. See Section 4.2 .

We note that $\mathrm{M}^{\natural}$-concavity in Lemma 3.3 is not required to show the if part, while it is required to show the only-if part. 
Let us define $f_{P}$ and $f_{Q}$ by

$$
f_{P}(x)=\sum_{i \in P} f_{i}\left(x_{(i)}\right), \quad f_{Q}(x)=\sum_{j \in Q} f_{j}\left(x_{(j)}\right) \quad\left(\forall x \in \mathbf{Z}^{E}\right) .
$$

Since $E_{(i)}$ and $E_{\left(i^{\prime}\right)}$ are disjoint for all $i, i^{\prime} \in P$ with $i \neq i^{\prime}$, function $f_{P}$ is $\mathrm{M}^{\natural}$ concave if all functions $f_{i}(i \in P)$ are $\mathrm{M}^{\natural}$-concave. Similarly, $f_{Q}$ is $\mathrm{M}^{\natural}$-concave if all functions $f_{j}(j \in Q)$ are. Moreover, the following assertions obviously hold.

Lemma 3.4: Condition (3.10) holds if and only if $x \in \arg \max \left\{f_{P}[+p](y) \mid y \leq\right.$ $\left.z_{P}\right\}$. Condition (3.11) holds if and only if $x \in \arg \max \left\{f_{Q}[-p](y) \mid y \leq z_{Q}\right\}$.

Furthermore, Assumption (A) is rewritten in terms of $f_{P}$ and $f_{Q}$ as:

$\left(\mathbf{A}^{\prime}\right)$ Effective domains $\operatorname{dom} f_{P}$ and $\operatorname{dom} f_{Q}$ are bounded and hereditary, and have the common minimum point $\mathbf{0} \in \mathbf{Z}^{E}$.

By Lemmas 3.3 and 3.4, Theorem 3.2 is a direct consequence of the following theorem.

Theorem 3.5: For $M^{\natural}$-concave functions $f_{P}, f_{Q}: \mathbf{Z}^{E} \rightarrow \mathbf{R} \cup\{-\infty\}$ satisfying $\left(\mathrm{A}^{\prime}\right)$ and for vectors $\underline{\pi} \in(\mathbf{R} \cup\{-\infty\})^{E}$ and $\bar{\pi} \in(\mathbf{R} \cup\{+\infty\})^{E}$ with $\underline{\pi} \leq \bar{\pi}$, there exist $x \in \mathbf{Z}^{E}, p \in \mathbf{R}^{E}$, and $z_{P}, z_{Q} \in(\mathbf{Z} \cup\{+\infty\})^{E}$ such that

$$
\begin{aligned}
& x \in \arg \max \left\{f_{P}[+p](y) \mid y \leq z_{P}\right\}, \\
& x \in \arg \max \left\{f_{Q}[-p](y) \mid y \leq z_{Q}\right\}, \\
& \underline{\pi} \leq p \leq \bar{\pi}, \\
& e \in E, z_{P}(e)<+\infty \Rightarrow p(e)=\underline{\pi}(e), z_{Q}(e)=+\infty, \\
& e \in E, z_{Q}(e)<+\infty \Rightarrow p(e)=\bar{\pi}(e), z_{P}(e)=+\infty .
\end{aligned}
$$

Moreover, if $f_{P}$ and $f_{Q}$ are integer-valued on their effective domains, $\underline{\pi} \in(\mathbf{Z} \cup$ $\{-\infty\})^{E}$, and $\bar{\pi} \in(\mathbf{Z} \cup\{+\infty\})^{E}$, then the above $p$ can be chosen from $\mathbf{Z}^{E}$.

We also say that a pair $(x, p)$ of $x \in \mathbf{Z}^{E}$ and $p \in \mathbf{R}^{E}$ is a pairwise strictly stable outcome if there exist $z_{P}, z_{Q} \in(\mathbf{Z} \cup\{+\infty\})^{E}$ satisfying (3.16) (3.20). We will give a proof of Theorem 3.5 in Section 4.3.

We next discuss relations between our model and existing models.

Remark 1: We briefly explain that our model includes the marriage model due to Gale and Shapley [12] and the assignment model due to Shapley and Shubik [26] as special cases. In these models, we are given pairs $\left(a_{i j}, b_{i j}\right) \in(\mathbf{R} \cup\{-\infty\})^{2}$ for all $(i, j) \in E=P \times Q$. Here $a_{i j}$ and $b_{i j}$ are interpreted as profits of $i$ and $j$ in the assignment model. Moreover, these define preferences in the marriage 
model: $i \in P$ prefers $j_{1}$ to $j_{2}$ if $a_{i j_{1}}>a_{i j_{2}}$, and $i$ is indifferent between $j_{1}$ and $j_{2}$ if $a_{i j_{1}}=a_{i j_{2}}$ (similarly, a preference of $j \in Q$ is defined by $b_{i j}(i \in P)$ ). We assume that $a_{i j}>0$ if $j$ is acceptable to $i$, and $a_{i j}=-\infty$ otherwise, and $b_{i j}>0$ if $i$ is acceptable to $j$, and $b_{i j}=-\infty$ otherwise. Define functions $f_{i}$ for all $i \in P$ and $f_{j}$ for all $j \in Q$ by

$$
\begin{aligned}
& f_{i}(x)=\left\{\begin{array}{cl}
a_{i j} & \text { if } x=\chi_{(i, j)} \text { for some } j \in Q \\
0 & \text { if } x=\mathbf{0} \\
-\infty & \text { otherwise }
\end{array}\right. \\
& f_{j}(x)=\left\{\begin{array}{cl}
b_{i j} & \text { if } x=\chi_{(i, j)} \text { for some } i \in P \\
0 & \text { if } x=\mathbf{0} \\
-\infty & \text { otherwise }
\end{array}\right.
\end{aligned}
$$

It can easily be shown that the above functions are $\mathrm{M}^{\natural}$-concave. We can show that, by putting $\underline{\pi}=\bar{\pi}=\mathbf{0}$, pairwise stability in our model coincides with pairwise stability in the marriage model. On the other hand, by putting $\underline{\pi}=(-\infty, \cdots,-\infty)$ and $\bar{\pi}=(+\infty, \cdots,+\infty)$, pairwise stability in our model coincides with pairwise stability in the assignment model. Furthermore, by Lemma 3.1, in these special cases, pairwise strict stability is identical with pairwise stability.

Remark 2: We briefly discuss the fact that Theorem 3.5 implies the existence of pairwise stable outcome in the model in Fujishige and Tamura [10]. This means that our model also includes many existing models (see Fujishige and Tamura [10] for details). In their model, two $\mathrm{M}^{\natural}$-concave functions $f_{P}, f_{Q}: \mathbf{Z}^{E} \rightarrow \mathbf{R} \cup\{-\infty\}$ satisfying $\left(\mathrm{A}^{\prime}\right)$, and an arbitrary partition $(F, R)$ of $E$ are given. For a vector $d$ on $E$ and $S \subseteq E$, let $\left.d\right|_{S}$ denote the restriction of $d$ on $S$. A vector $x \in \operatorname{dom} f_{P} \cap \operatorname{dom} f_{Q}$ is called an $f_{P} f_{Q}$-pairwise stable solution with respect to $(F, R)$ if there exist $p \in \mathbf{R}^{E}$, disjoint subsets $R_{P}$ and $R_{Q}$ of $R, \hat{z}_{P} \in \mathbf{Z}^{R_{P}}$, and $\hat{z}_{Q} \in \mathbf{Z}^{R_{Q}}$ such that

$$
\begin{aligned}
\left.p\right|_{R} & =\mathbf{0}, \\
x & \in \arg \max \left\{f_{P}[+p](y)|y|_{R_{P}} \leq \hat{z}_{P}\right\}, \\
x & \in \arg \max \left\{f_{Q}[-p](y)|y|_{R_{Q}} \leq \hat{z}_{Q}\right\} .
\end{aligned}
$$

We can show that $f_{P} f_{Q}$-pairwise stability is equivalent to our pairwise strict stability in the case where $\underline{\pi}(e)=\bar{\pi}(e)=0$ for all $e \in R$, and $\underline{\pi}(e)=-\infty$ and $\bar{\pi}(e)=+\infty$ for all $e \in F$.

We finally give one illustration of our model.

Example 4: We consider the problem of allocating dance partners between set $P=\left\{m_{1}, m_{2}\right\}$ of two men and set $Q=\left\{w_{1}, w_{2}\right\}$ of two women. Here $E=P \times Q$. 
We abbreviate pair $\left(m_{i}, w_{j}\right)$ by $(i, j)$ and denote the $(i, j)$-component of a vector $d$ by $d_{i j}$. Think of a situation when $m_{1}$ and $w_{1}$ are professional and the others are amateur, and $m_{2}$ and $w_{1}$ are a married couple. Because a lesson fee (which should be nonnegative) is allowed between a professional and an amateur, and because a lesson fee between husband and wife is meaningless, we set

$$
\underline{\pi}=\mathbf{0}, \quad\left(\bar{\pi}_{11}, \bar{\pi}_{12}, \bar{\pi}_{21}, \bar{\pi}_{22}\right)=(0,+\infty, 0,0) .
$$

We assume that they have the following preferences:

- everyone wants to dance as many times as possible, up to four times,

- $m_{1}$ prefers $w_{1}$ to $w_{2}$,

- $w_{1}$ and $w_{2}$ are indifferent for $m_{2}$,

- every woman wants to dance with $m_{1}$ and $m_{2}$ as equally as possible.

Denoting by $x_{i j}$ the number of times $m_{i}$ and $w_{j}$ dance together, we further assume that their preferences are described by the following four utility functions:

$$
\begin{aligned}
f_{m_{1}}\left(x_{11}, x_{12}\right) & = \begin{cases}10 x_{11}+8 x_{12} & \text { if } 0 \leq x_{11}+x_{12} \leq 4 \\
-\infty & \text { otherwise, }\end{cases} \\
f_{m_{2}}\left(x_{21}, x_{22}\right) & = \begin{cases}10 x_{21}+10 x_{22} & \text { if } 0 \leq x_{21}+x_{22} \leq 4 \\
-\infty & \text { otherwise, }\end{cases} \\
f_{w_{1}}\left(x_{11}, x_{21}\right) & = \begin{cases}10 x_{11}+10 x_{21}-\left(x_{11}^{2}+x_{21}^{2}\right) / 2 & \text { if } 0 \leq x_{11}+x_{21} \leq 4 \\
-\infty & \text { otherwise, }\end{cases} \\
f_{w_{2}}\left(x_{12}, x_{22}\right) & = \begin{cases}10 x_{12}+10 x_{22}-\left(x_{12}^{2}+x_{22}^{2}\right) / 2 & \text { if } 0 \leq x_{12}+x_{22} \leq 4 \\
-\infty & \text { otherwise, }\end{cases}
\end{aligned}
$$

which are used in Fujishige and Tamura [10, Example 4]. We note that the above functions are $\mathrm{M}^{\natural}$-concave, because these are defined by laminar families. Thus, the problem is formulated as our model. We have the following pairwise strictly stable outcomes, $x$ and the range of $s_{12}$, together with $z_{P}$ and $z_{Q}$ :

\begin{tabular}{c|c|c|c}
$\left(x_{11}, x_{12}, x_{21}, x_{22}\right)$ & range of $s_{12}$ & $z_{P}$ & $z_{Q}$ \\
\hline$(3,1,1,3)$ & {$[1,2]$} & $(3,-,-,-)$ & $(-,-, 1,-)$ \\
$(2,2,2,2)$ & {$[0,2]$} & $(2,-,-,-)$ & $(-,-,-, 2)$ \\
$(1,3,3,1)$ & {$[2,2]$} & $(-,-,-,-)$ & $(1,-,-, 1)$ \\
$(0,4,4,0)$ & {$[2,6.5]$} & $(-,-,-,-)$ & $(0,-,-, 0)$
\end{tabular}

where - means $+\infty$ in the above table. By setting $p=\left(0, s_{12}, 0,0\right)$ and by using $z_{P}$ and $z_{Q}$ given above, we can easily check the pairwise strict stability of the above outcomes, due to Lemma 3.3. Lemma 3.3 gives a good characterization of pairwise strictly stable outcomes. 


\section{Proofs}

In this section we give proofs of Lemma 3.1, Lemma 3.3, and Theorem 3.5.

\subsection{A proof of Lemma 3.1}

It is enough to show that if an outcome $(x, s)$ is pairwise quasi-unstable, then it is also pairwise unstable. Let $(x, s)$ be a pairwise quasi-unstable outcome. We may assume that $(x, s)$ satisfies incentive constraints (3.1) and (3.2). Then there exist $i \in P, j \in Q, \alpha \in[\underline{\pi}(i, j), \bar{\pi}(i, j)], y^{\prime} \in \mathbf{Z}^{E_{(i)}}$ and $y^{\prime \prime} \in \mathbf{Z}^{E_{(j)}}$ satisfying (3.3) (3.6).

We first deal with Case (i). In this case, $s(i, j)=\alpha$ holds. Because $(x, s)$ satisfies incentive constraints, we have $y^{\prime}(i, j)>x(i, j)$ and $y^{\prime \prime}(i, j)>x(i, j)$. In addition, we assume that $y^{\prime}(i, j)$ and $y^{\prime \prime}(i, j)$, respectively, are as small as possible among vectors satisfying $(3.3) \sim(3.6)$. By $\left(\mathrm{M}^{\natural}\right)$ for $y^{\prime}$ and $x_{(i)}$, there exists $e^{\prime} \in$ $\operatorname{supp}^{-}\left(y^{\prime}-x_{(i)}\right) \cup\{0\}$ such that, putting $e=(i, j)$,

$$
\begin{aligned}
& f_{i}\left[+\left(s_{(i)}^{-j}, \alpha\right)\right]\left(y^{\prime}\right)+f_{i}\left[+s_{(i)}\right]\left(x_{(i)}\right) \\
= & f_{i}\left[+\left(s_{(i)}^{-j}, \alpha\right)\right]\left(y^{\prime}\right)+f_{i}\left[+\left(s_{(i)}^{-j}, \alpha\right)\right]\left(x_{(i)}\right) \\
\leq & f_{i}\left[+\left(s_{(i)}^{-j}, \alpha\right)\right]\left(y^{\prime}-\chi_{e}+\chi_{e^{\prime}}\right)+f_{i}\left[+\left(s_{(i)}^{-j}, \alpha\right)\right]\left(x_{(i)}+\chi_{e}-\chi_{e^{\prime}}\right) .
\end{aligned}
$$

Since $f_{i}\left[+\left(s_{(i)}^{-j}, \alpha\right)\right]\left(y^{\prime}\right)>f_{i}\left[+\left(s_{(i)}^{-j}, \alpha\right)\right]\left(y^{\prime}-\chi_{e}+\chi_{e^{\prime}}\right)$ by the choice of $y^{\prime}$, we obtain $f_{i}\left[+s_{(i)}\right]\left(x_{(i)}\right)<f_{i}\left[+\left(s_{(i)}^{-j}, \alpha\right)\right]\left(x_{(i)}+\chi_{e}-\chi_{e^{\prime}}\right)$. This implies that $y^{\prime}(i, j)=x(i, j)+1$, since $x_{(i)}+\chi_{e}-\chi_{e^{\prime}}$ is a possible candidate for $y^{\prime}$. Analogously, we can show that $y^{\prime \prime}(i, j)=x(i, j)+1$. Hence $y^{\prime}$ and $y^{\prime \prime}$ also satisfy (3.7).

We next consider Cases (ii) and (iii). We assume that $s(i, j)<\alpha$ (The case where $s(i, j)=\alpha$ can be treated similarly as in Case (i) and we can also deal with the case where $s(i, j)>\alpha$ similarly as shown below). If $x(i, j)=0$, then we can show the assertion in the same way as in Case (i). Hence we assume that $x(i, j)>0$. In this case, the following relations

$$
\begin{aligned}
f_{i}\left[+s_{(i)}\right]\left(x_{(i)}\right) & <f_{i}\left[+\left(s_{(i)}^{-j}, \alpha\right)\right]\left(x_{(i)}\right), \\
f_{j}\left[-s_{(j)}\right](y) & \geq f_{j}\left[-\left(s_{(j)}^{-i}, \alpha\right)\right](y) \quad\left(\forall y \leq x_{(j)}\right)
\end{aligned}
$$

hold. Thus, $y^{\prime \prime}(i, j)$ must be greater than $x(i, j)$, so that $y^{\prime \prime}(i, j) \geq 2$. Therefore, it is sufficient to deal with Case (iii) only. Replace $y^{\prime}$ by $x_{(i)}+\left(y^{\prime \prime}(i, j)-x(i, j)\right) \chi_{(i, j)}$, which belongs to $\operatorname{dom} f_{i}$. We then have $f_{i}\left[+\left(s_{(i)}^{-j}, \alpha\right)\right]\left(y^{\prime}\right)>f_{i}\left[+s_{(i)}\right]\left(y^{\prime}\right)$. Since $x(i, j)>0, f_{i}$ is linear over $\operatorname{dom} f_{i}$, and $(x, s)$ satisfies incentive constraints, we also have $f_{i}\left[+s_{(i)}\right]\left(y^{\prime}\right) \geq f_{i}\left[+s_{(i)}\right]\left(x_{(i)}\right)$. Hence (3.3), (3.4), and (3.7) are satisfied by the new $y^{\prime}$. 


\subsection{A proof of Lemma 3.3}

The only-if part: Let $(x, s)$ be a pairwise strictly stable outcome. For each pair $(i, j) \in E$ with $x(i, j)=0$, we define $r(i, j)$ as the supremum of the set of $\alpha$ 's satisfying (3.8) without the constraint $\alpha \leq \bar{\pi}(i, j)$. (We have $r(i, j) \neq+\infty$ if there exists $y \in \operatorname{dom} f_{i}$ such that $y(i, j)>0$ and $y\left(i, j^{\prime}\right) \leq x\left(i, j^{\prime}\right)$ for all $j^{\prime} \in Q \backslash\{j\}$.) If $r(i, j)=+\infty$, then we redefine $r(i, j)$ as the infimum of the set of $\alpha$ 's satisfying (3.9) without the constraint $\underline{\pi}(i, j) \leq \alpha$. (We have $r(i, j) \neq-\infty$ if there exists $y \in \operatorname{dom} f_{j}$ such that $y(i, j)>0$ and $y\left(i^{\prime}, j\right) \leq x\left(i^{\prime}, j\right)$ for all $i^{\prime} \in P \backslash\{i\}$.) If $r(i, j)=-\infty$, then we further redefine $r(i, j)=-b$ for a sufficiently large positive number $b$. Then, we define $p \in \mathbf{R}^{E}$ by

$$
p(i, j)=\left\{\begin{array}{ll}
s(i, j) & \text { if } x(i, j)>0 \\
r(i, j) & \text { else if } \underline{\pi}(i, j) \leq r(i, j) \leq \bar{\pi}(i, j) \\
\underline{\pi}(i, j) & \text { else if } r(i, j)<\underline{\pi}(i, j) \\
\bar{\pi}(i, j) & \text { else if } \bar{\pi}(i, j)<r(i, j)
\end{array} \quad(\forall(i, j) \in E) .\right.
$$

Condition (3.12) is satisfied by $p$ because $s$ is $x$-compatible.

We also define $z_{P}$ and $z_{Q}$ by

$$
\begin{aligned}
& z_{P}(i, j)=\left\{\begin{array}{ll}
x(i, j) & \text { if }(3.8) \text { does not hold for } \alpha=p(i, j) \\
+\infty & \text { otherwise }
\end{array} \quad(\forall(i, j) \in E),\right. \\
& z_{Q}(i, j)=\left\{\begin{array}{ll}
x(i, j) & \text { if }(3.9) \text { does not hold for } \alpha=p(i, j) \\
+\infty & \text { otherwise }
\end{array} \quad(\forall(i, j) \in E) .\right.
\end{aligned}
$$

It follows from the pairwise strict stability of $(x, s)$ that $z_{P}(i, j)=+\infty$ or $z_{Q}(i, j)=$ $+\infty$ holds. We consider the case where $z_{P}(i, j)<+\infty$. In this case, there exists $y^{\prime} \in \mathbf{Z}^{E_{(i)}}$ such that $f_{i}\left[+p_{(i)}\right]\left(x_{(i)}\right)<f_{i}\left[+p_{(i)}\right]\left(y^{\prime}\right)$ and $y^{\prime}\left(i, j^{\prime}\right) \leq x\left(i, j^{\prime}\right)$ for all $j^{\prime} \in Q \backslash\{j\}$, where note that $f_{i}\left[+p_{(i)}\right]\left(x_{(i)}\right)=f_{i}\left[+s_{(i)}\right]\left(x_{(i)}\right)$ and $f_{i}\left[+p_{(i)}\right]\left(y^{\prime}\right)=$ $f_{i}\left[+\left(s_{(i)}^{-j}, p(i, j)\right)\right]\left(y^{\prime}\right)$. We show (3.13). Suppose, to the contrary, that $p(i, j)>$ $\underline{\pi}(i, j)$. If $x(i, j)>0$, then for a sufficiently small number $\epsilon>0$ we have

$$
\begin{aligned}
f_{i}\left[+p_{(i)}\right]\left(x_{(i)}\right) & <f_{i}\left[+\left(p_{(i)}-\epsilon \chi_{(i, j)}\right)\right]\left(y^{\prime}\right), \\
f_{j}\left[-p_{(j)}\right]\left(x_{(j)}\right) & <f_{j}\left[-\left(p_{(j)}-\epsilon \chi_{(i, j)}\right)\right]\left(x_{(j)}\right),
\end{aligned}
$$

which imply that neither (3.8) nor (3.9) holds for $\alpha=p(i, j)-\epsilon \geq \underline{\pi}(i, j)$, a contradiction. Hence, assume $x(i, j)=0$. Since $p(i, j)>\underline{\pi}(i, j)$, we have $p(i, j) \leq$ $r(i, j)$ by (4.1). As the right-hand side of (3.8) is nondecreasing in $\alpha$ and $p(i, j) \leq$ $r(i, j)$, the definition of $r(i, j)$ guarantees that (3.8) holds for $\alpha=p(i, j)$. However, this contradicts $z_{P}(i, j)<+\infty$. Thus, we have (3.13).

We next consider the case where $z_{Q}(i, j)<+\infty$ and show (3.14). Suppose, to the contrary, that $p(i, j)<\bar{\pi}(i, j)$. If $x(i, j)>0$, then we reach a contradiction in 
the same way as above. Hence assume $x(i, j)=0$. By (4.1) and the assumption we have $p(i, j) \geq r(i, j)$. If $r(i, j)$ is defined by (3.8), then for a sufficiently small number $\epsilon>0$, neither (3.8) nor (3.9) holds for $\alpha=p(i, j)+\epsilon \leq \bar{\pi}(i, j)$, which is a contradiction. In the other case (where $r(i, j)$ is either defined by (3.9) or sufficiently small $-b)$, (3.9) holds for $\alpha=p(i, j)$ since $p(i, j) \geq r(i, j)$. However, this contradicts the assumption that $z_{Q}(i, j)<+\infty$. Hence, we have (3.14).

We will finally show (3.10) ((3.11) can be shown similarly). Suppose that (3.10) does not hold, i.e., for some $i \in P$ there exists $y^{\prime} \in \arg \max \left\{f_{i}\left[+p_{(i)}\right](y) \mid y \leq z_{(i)}\right\}$ with $f_{i}\left[+p_{(i)}\right]\left(x_{(i)}\right)<f_{i}\left[+p_{(i)}\right]\left(y^{\prime}\right)$. We choose $y^{\prime} \in \arg \max \left\{f_{i}\left[+p_{(i)}\right](y) \mid y \leq z_{(i)}\right\}$ with $f_{i}\left[+p_{(i)}\right]\left(x_{(i)}\right)<f_{i}\left[+p_{(i)}\right]\left(y^{\prime}\right)$ that minimizes $\sum\left\{y^{\prime}(e)-x_{(i)}(e) \mid e \in \operatorname{supp}^{+}\left(y^{\prime}-\right.\right.$ $\left.\left.x_{(i)}\right)\right\}$. Since $f_{i}\left[+s_{(i)}\right](y)=f_{i}\left[+p_{(i)}\right](y)$ holds for all $y \in \mathbf{Z}^{E_{(i)}}$ with $\mathbf{0} \leq y \leq x_{(i)}$, (3.1) implies the existence of $e \in E_{(i)}$ with $y^{\prime}(e)>x_{(i)}(e)$. By $\left(\mathrm{M}^{\natural}\right)$, there exists $e^{\prime} \in \operatorname{supp}^{-}\left(y^{\prime}-x_{(i)}\right) \cup\{0\}$ such that

$$
f_{i}\left[+p_{(i)}\right]\left(y^{\prime}\right)+f_{i}\left[+p_{(i)}\right]\left(x_{(i)}\right) \leq f_{i}\left[+p_{(i)}\right]\left(y^{\prime}-\chi_{e}+\chi_{e^{\prime}}\right)+f_{i}\left[+p_{(i)}\right]\left(x_{(i)}+\chi_{e}-\chi_{e^{\prime}}\right) .
$$

By the definition of $y^{\prime}$, we have

$$
f_{i}\left[+p_{(i)}\right]\left(y^{\prime}\right)>f_{i}\left[+p_{(i)}\right]\left(y^{\prime}-\chi_{e}+\chi_{e^{\prime}}\right)
$$

The above two inequalities imply $f_{i}\left[+p_{(i)}\right]\left(x_{(i)}\right)<f_{i}\left[+p_{(i)}\right]\left(x_{(i)}+\chi_{e}-\chi_{e^{\prime}}\right)$, which yields that $z_{P}(e)=x_{(i)}(e)$, by (4.2). This contradicts $y^{\prime} \leq z_{(i)}$. Hence (3.10) holds.

The if part: $\quad$ Let $p \in \mathbf{R}^{E}$ and $z_{P}, z_{Q} \in(\mathbf{Z} \cup\{+\infty\})^{E}$ be vectors satisfying (3.10) (3.14). We define a vector $s \in \mathbf{R}^{E}$ by

$$
s(i, j)=\left\{\begin{array}{ll}
p(i, j) & \text { if } x(i, j)>0 \\
0 & \text { if } x(i, j)=0
\end{array} \quad(\forall(i, j) \in E) .\right.
$$

It follows from (3.12) that $s$ is $x$-compatible. We will show that $(x, s)$ is pairwise strictly stable. Since $x_{(k)} \leq z_{(k)}$ for all $k \in P \cup Q$, Conditions (3.1) and (3.2) are direct consequences of (3.10) and (3.11). Suppose, to the contrary, that there exist $i \in P, j \in Q, \alpha \in[\underline{\pi}(i, j), \bar{\pi}(i, j)], y^{\prime} \in \mathbf{Z}^{E_{(i)}}$ and $y^{\prime \prime} \in \mathbf{Z}^{E_{(j)}}$ such that

$$
\begin{aligned}
& f_{i}\left[+s_{(i)}\right]\left(x_{(i)}\right)<f_{i}\left[+\left(s_{(i)}^{-j}, \alpha\right)\right]\left(y^{\prime}\right), \\
& y^{\prime}\left(i, j^{\prime}\right) \leq x\left(i, j^{\prime}\right) \quad\left(\forall j^{\prime} \in Q \backslash\{j\}\right),
\end{aligned}
$$

and

$$
\begin{aligned}
& f_{j}\left[-s_{(j)}\right]\left(x_{(j)}\right)<f_{j}\left[-\left(s_{(j)}^{-i}, \alpha\right)\right]\left(y^{\prime \prime}\right), \\
& y^{\prime \prime}\left(i^{\prime}, j\right) \leq x\left(i^{\prime}, j\right) \quad\left(\forall i^{\prime} \in P \backslash\{i\}\right) .
\end{aligned}
$$

Definition (4.4) yields that

$$
f_{i}\left[+s_{(i)}\right]\left(x_{(i)}\right)=f_{i}\left[+p_{(i)}\right]\left(x_{(i)}\right), \quad f_{i}\left[+\left(s_{(i)}^{-j}, \alpha\right)\right]\left(y^{\prime}\right)=f_{i}\left[+\left(p_{(i)}^{-j}, \alpha\right)\right]\left(y^{\prime}\right) .
$$


Hence, by (3.10) and since $y^{\prime} \geq \mathbf{0}$, Condition (4.5) implies that either (Case 1) $y^{\prime}(i, j)>z_{(i)}(i, j)$ or $($ Case 2$) y^{\prime}(i, j) \leq z_{(i)}(i, j)$ and $p(i, j)<\alpha$. Similarly, by (3.11) and (4.6), we have either (Case 3) $y^{\prime \prime}(i, j)>z_{(j)}(i, j)$ or (Case 4) $y^{\prime \prime}(i, j) \leq z_{(j)}(i, j)$ and $\alpha<p(i, j)$. Trivially, (Case 2) and (Case 4) are inconsistent. By (3.13) or (3.14), (Case 1) and (Case 3) do not hold simultaneously. Also, (Case 1) together with (3.13) implies $p(i, j)=\underline{\pi}(i, j)$, which is irreconcilable with (Case 4). Analogously, (Case 2) is irreconcilable with (Case 3), due to (3.14). This means that (4.5) and (4.6) do not hold simultaneously, a contradiction. Hence $(x, s)$ is pairwise strictly stable.

\subsection{A proof of Theorem 3.5}

We assume that given $\mathrm{M}^{\natural}$-concave functions $f_{P}, f_{Q}: \mathbf{Z}^{E} \rightarrow \mathbf{R} \cup\{-\infty\}$ satisfy Assumption $\left(\mathrm{A}^{\prime}\right)$.

Before describing our constructive proof, we give several known results on $\mathrm{M}^{\natural}$ concave functions. For an $\mathrm{M}^{\natural}$-concave function $f: \mathbf{Z}^{E} \rightarrow \mathbf{R} \cup\{-\infty\}$, we define $\hat{f}: \mathbf{Z}^{\{0\} \cup E} \rightarrow \mathbf{R} \cup\{-\infty\}$ by

$$
\hat{f}\left(y_{0}, y\right)=\left\{\begin{array}{ll}
f(y) & \text { if } y_{0}=-y(E) \\
-\infty & \text { otherwise }
\end{array} \quad\left(\forall\left(y_{0}, y\right) \in \mathbf{Z}^{\{0\} \cup E}\right)\right.
$$

where $0 \notin E$ and $y(E)$ denotes the sum of all the components of $y$. Function $\hat{f}$ is called an $M$-concave function (Murota $[18,19]$ ). Let $\tilde{E}=\{0\} \cup E$. For each vector $x \in \mathbf{R}^{E}$ we denote by $\hat{x}$ the vector $(-x(E), x) \in \mathbf{R}^{\tilde{E}}$, and we write vectors in $\mathbf{R}^{\tilde{E}}$ by putting tildes such as $\tilde{p}$ and $\tilde{y}$ (e.g., $\tilde{\chi}_{e}$ is the characteristic vector of $e$ on $\tilde{E}$ ). An M-convex function can be characterized by the following exchange property (Murota [18, 19]):

(M) $\forall \tilde{x}, \tilde{y} \in \operatorname{dom} \hat{f}, \forall e \in \operatorname{supp}^{+}(\tilde{x}-\tilde{y}), \exists e^{\prime} \in \operatorname{supp}^{-}(\tilde{x}-\tilde{y}):$

$$
\hat{f}(\tilde{x})+\hat{f}(\tilde{y}) \leq \hat{f}\left(\tilde{x}-\tilde{\chi}_{e}+\tilde{\chi}_{e^{\prime}}\right)+\hat{f}\left(\tilde{y}+\tilde{\chi}_{e}-\tilde{\chi}_{e^{\prime}}\right) .
$$

((M) is written as (-M-EXC) in Murota [20].) In particular, an M-concave function is also $\mathrm{M}^{\natural}$-concave. For a vector $\tilde{p}=\left(p_{0}, p\right) \in \mathbf{R}^{\tilde{E}}$ we have

$$
x \in \arg \max f\left[p-p_{0} \mathbf{1}\right] \Longleftrightarrow \hat{x} \in \arg \max \hat{f}[\tilde{p}] .
$$

The maximizers of an M-concave function have a good characterization as follows.

Theorem 4.1 (Murota $[18,19]$ ): For an M-concave function $\hat{f}: \mathbf{Z}^{\tilde{E}} \rightarrow \mathbf{R} \cup\{-\infty\}$ and $\tilde{x} \in \operatorname{dom} \hat{f}$, we have $\tilde{x} \in \arg \max \hat{f}$ if and only if $\hat{f}(\tilde{x}) \geq \hat{f}\left(\tilde{x}-\tilde{\chi}_{e}+\tilde{\chi}_{e^{\prime}}\right)$ for all e, $e^{\prime} \in \tilde{E}$. 
The following property is a direct consequence of (M).

Lemma 4.2: Let $\hat{f}$ be an $M$-concave function. Then, for any $\tilde{x}, \tilde{y} \in \arg \max \hat{f}$ and for any $e \in \operatorname{supp}^{+}(\tilde{x}-\tilde{y})$, there exists $e^{\prime} \in \operatorname{supp}^{-}(\tilde{x}-\tilde{y})$ such that $\tilde{x}-\tilde{\chi}_{e}+\tilde{\chi}_{e^{\prime}}$, $\tilde{y}+\tilde{\chi}_{e}-\tilde{\chi}_{e^{\prime}} \in \arg \max \hat{f}$.

A set $B$ of integer vectors satisfying the property in Lemma 4.2 is called an $M$ convex set, which is a so-called base polyhedron in $\mathbf{Z}^{\tilde{E}}$. M-convex sets have the following property.

Lemma 4.3 (Lemma 4.5 in Fujishige [9]): Let $B$ be an $M$-convex set. For any $\tilde{x} \in B$ and any distinct $e_{1}, e_{1}^{\prime}, e_{2}, e_{2}^{\prime}, \cdots, e_{r}, e_{r}^{\prime} \in \tilde{E}$, if $\tilde{x}-\tilde{\chi}_{e_{i}}+\tilde{\chi}_{e_{i}^{\prime}} \in B$ for all $i=$ $1, \cdots, r$ and $\tilde{x}-\tilde{\chi}_{e_{i}}+\tilde{\chi}_{e_{j}^{\prime}} \notin B$ for all $i, j$ with $i<j$, then $\tilde{y}=\tilde{x}-\sum_{i=1}^{r}\left(\tilde{\chi}_{e_{i}}-\tilde{\chi}_{e_{i}^{\prime}}\right) \in B$.

Lemma 4.4 (Fujishige and Tamura [10]): Let $\hat{f}: \mathbf{Z}^{\tilde{E}} \rightarrow \mathbf{R} \cup\{-\infty\}$ be an $M$ concave function. For an element $e \in \tilde{E}$ let $\tilde{z}_{1}, \tilde{z}_{2} \in(\mathbf{Z} \cup\{+\infty\})^{\tilde{E}}$ be vectors such that $\tilde{z}_{1}=\tilde{z}_{2}+\tilde{\chi}_{e}, \arg \max \left\{\hat{f}(\tilde{y}) \mid \tilde{y} \leq \tilde{z}_{1}\right\} \neq \emptyset$, and $\arg \max \left\{\hat{f}(\tilde{y}) \mid \tilde{y} \leq \tilde{z}_{2}\right\} \neq \emptyset$. Then, the following two statements hold:

(a) For each $\tilde{x} \in \arg \max \left\{\hat{f}(\tilde{y}) \mid \tilde{y} \leq \tilde{z}_{1}\right\}$ there exists $e^{\prime} \in \tilde{E}\left(\right.$ possibly $\left.e^{\prime}=e\right)$ such that

$$
\tilde{x}-\tilde{\chi}_{e}+\tilde{\chi}_{e^{\prime}} \in \arg \max \left\{\hat{f}(\tilde{y}) \mid \tilde{y} \leq \tilde{z}_{2}\right\} .
$$

(b) For each $\tilde{x} \in \arg \max \left\{\hat{f}(\tilde{y}) \mid \tilde{y} \leq \tilde{z}_{2}\right\}$ there exists $e^{\prime} \in \tilde{E}$ (possibly $\left.e^{\prime}=e\right)$ such that

$$
\tilde{x}+\tilde{\chi}_{e}-\tilde{\chi}_{e^{\prime}} \in \arg \max \left\{\hat{f}(\tilde{y}) \mid \tilde{y} \leq \tilde{z}_{1}\right\} .
$$

Lemma 4.5 (Fujishige and Tamura [10]): For an M-concave function $\hat{f}: \mathbf{Z}^{\tilde{E}} \rightarrow$ $\mathbf{R} \cup\{-\infty\}$ and a vector $\tilde{z}_{2} \in(\mathbf{Z} \cup\{+\infty\})^{\tilde{E}}$ suppose that $\arg \max \left\{\hat{f}(\tilde{y}) \mid \tilde{y} \leq \tilde{z}_{2}\right\} \neq$ $\emptyset$. For any $\tilde{x} \in \arg \max \left\{\hat{f}(\tilde{y}) \mid \tilde{y} \leq \tilde{z}_{2}\right\}$ and any $\tilde{z}_{1} \in(\mathbf{Z} \cup\{+\infty\})^{\tilde{E}}$ such that (i) $\tilde{z}_{1} \geq \tilde{z}_{2}$ and (ii) if $\tilde{x}(e)=\tilde{z}_{2}(e)$ then $\tilde{z}_{1}(e)=\tilde{z}_{2}(e)$, we have $\tilde{x} \in \arg \max \{\hat{f}(\tilde{y}) \mid$ $\left.\tilde{y} \leq \tilde{z}_{1}\right\}$.

By relation (4.7) between $\mathrm{M}^{\natural}$-concave and M-concave functions, the problem of finding a pairwise strictly stable outcome is equivalent to that of finding $\hat{x}_{P}, \hat{x}_{Q} \in$ $\mathbf{Z}^{\tilde{E}}, \tilde{p}=\left(p_{0}, p\right) \in \mathbf{R}^{\tilde{E}}$ and $\tilde{z}_{P}, \tilde{z}_{Q} \in(\mathbf{Z} \cup\{+\infty\})^{\tilde{E}}$ such that

$$
\begin{aligned}
& \hat{x}_{P}=\hat{x}_{Q}, \\
& \hat{x}_{P} \in \arg \max \left\{\hat{f}_{P}[+\tilde{p}](\tilde{y}) \mid \tilde{y} \leq \tilde{z}_{P}\right\}, \\
& \hat{x}_{Q} \in \arg \max \left\{\hat{f}_{Q}[-\tilde{p}](\tilde{y}) \mid \tilde{y} \leq \tilde{z}_{Q}\right\}, \\
& p_{0}=0, \quad \underline{\pi} \leq p \leq \bar{\pi} \\
& \tilde{z}_{P}(0)=+\infty,\left[e \in E, \tilde{z}_{P}(e)<+\infty \Rightarrow \tilde{p}(e)=\underline{\pi}(e), \tilde{z}_{Q}(e)=+\infty\right], \\
& \tilde{z}_{Q}(0)=+\infty,\left[e \in E, \tilde{z}_{Q}(e)<+\infty \Rightarrow \tilde{p}(e)=\bar{\pi}(e), \tilde{z}_{P}(e)=+\infty\right] .
\end{aligned}
$$


In the sequel, we show an algorithm that finds $\hat{x}_{P}, \hat{x}_{Q}, \tilde{p}, \tilde{z}_{P}$, and $\tilde{z}_{Q}$ satisfying (4.8) (4.13). Initially, we put $\tilde{p}$ as:

$$
\tilde{p}(e):=\left\{\begin{array}{cl}
0 & \text { if } e=0 \\
\bar{\pi}(e) & \text { if } \bar{\pi}(e)<+\infty \\
b & \text { otherwise }
\end{array} \quad(\forall e \in \tilde{E}),\right.
$$

where $b$ is a sufficiently large positive integer to be specified later. Furthermore, put $\tilde{z}_{P}:=(+\infty, \cdots,+\infty)$ and choose any $\hat{x}_{P} \in \arg \max \hat{f}_{P}[+\tilde{p}]$. Obviously, (4.9), (4.11), and (4.12) are satisfied. We define $\tilde{z}_{Q}$ by

$$
\tilde{z}_{Q}(e)=\left\{\begin{array}{cl}
+\infty & \text { if } e=0 \\
\hat{x}_{P}(e) & \text { if } \bar{\pi}(e)<+\infty \\
+\infty & \text { otherwise }
\end{array} \quad(\forall e \in \tilde{E})\right.
$$

and choose an $\hat{x}_{Q}$ satisfying (4.10). Condition (4.13) evidently holds. Moreover, by setting $b$ to be a large enough integer so that $\hat{x}_{Q}(e)=0$ for all $e \in E$ with $\bar{\pi}(e)=+\infty$, we have

$$
\hat{x}_{Q}(e) \leq \hat{x}_{P}(e) \quad(\forall e \in E) .
$$

From Assumption $\left(\mathrm{A}^{\prime}\right)$, such a $b$ exists. By Lemma 4.5, (4.10) is preserved even if $\tilde{z}_{Q}(e)$ is set to $+\infty$ for every $e \in E$ with $\tilde{p}(e)=\bar{\pi}(e)$ and $\hat{x}_{Q}(e)<\hat{x}_{P}(e)$. Thus we can assume that the following condition is satisfied:

$$
e \in E, \tilde{z}_{Q}(e)<+\infty \Rightarrow \hat{x}_{Q}(e)=\hat{x}_{P}(e)=\tilde{z}_{Q}(e) .
$$

Our aim is to modify vectors $\hat{x}_{P}, \hat{x}_{Q}, \tilde{p}, \tilde{z}_{P}$, and $\tilde{z}_{Q}$ preserving $(4.9) \sim(4.15)$ and eventually to attain (4.8).

We now assume that we are given vectors $\hat{x}_{P}, \hat{x}_{Q}, \tilde{p}, \tilde{z}_{P}$ and $\tilde{z}_{Q}$ satisfying (4.9) (4.15) but not (4.8). Let $L$ and $U$ be subsets of $E$ defined by

$$
\begin{aligned}
L & =\{e \in E \mid \tilde{p}(e)=\underline{\pi}(e)\} \\
U & =\left\{e \in E \mid \tilde{z}_{Q}(e)<+\infty\right\} .
\end{aligned}
$$

It follows from (4.13) and (4.15) that for all $e \in U$ we have $\tilde{p}(e)=\bar{\pi}(e)$ and $\hat{x}_{Q}(e)=\hat{x}_{P}(e)=\tilde{z}_{Q}(e)$. Note that $L$ and $U$ may have a common element $e$ with $\underline{\pi}(e)=\bar{\pi}(e)$.

We divide our argument into two parts that treat: (Case 1) there exists $e \in L$ with $\hat{x}_{Q}(e)<\hat{x}_{P}(e)$; (Case 2) the other case.

In (Case 1 ), we will modify $\hat{x}_{P}, \hat{x}_{Q}, \tilde{z}_{P}$ and $\tilde{z}_{Q}$ while keeping $\tilde{p}$ the same. Let $e$ be an element of $L$ with $\hat{x}_{Q}(e)<\hat{x}_{P}(e)$. From (4.15), we have $\tilde{z}_{Q}(e)=+\infty$, and hence, we can assume $\tilde{z}_{P}(e)=\hat{x}_{P}(e)$ while preserving (4.9) and (4.12). We replace $\tilde{z}_{P}(e)$ by $\hat{x}_{P}(e)-1$. By (a) of Lemma 4.4 , there exists $e^{\prime} \in \tilde{E} \backslash\{e\}$ such that 
$\hat{x}_{P}:=\hat{x}_{P}-\tilde{\chi}_{e}+\tilde{\chi}_{e^{\prime}}$ satisfies $(4.9)$ for the modified $\tilde{z}_{P}$. If $\tilde{z}_{Q}\left(e^{\prime}\right)=+\infty$ then $\hat{x}_{Q}$ and $\tilde{z}_{Q}$ are kept the same, and Conditions $(4.9) \sim(4.15)$ are preserved. In the case where $\tilde{z}_{Q}\left(e^{\prime}\right)<+\infty$ (i.e., $e^{\prime} \in U$ ), we modify $\hat{x}_{Q}$ and $\tilde{z}_{Q}$ as follows. By (4.15), for the updated $\hat{x}_{P}$ we have $\hat{x}_{P}\left(e^{\prime}\right)=\hat{x}_{Q}\left(e^{\prime}\right)+1$. Thus, (b) of Lemma 4.4 guarantees the existence of $e^{\prime \prime} \in E$, which may coincide with $e^{\prime}$, such that $\hat{x}_{Q}:=\hat{x}_{Q}+\tilde{\chi}_{e^{\prime}}-\tilde{\chi}_{e^{\prime \prime}}$ and $\tilde{z}_{Q}:=\tilde{z}_{Q}+\tilde{\chi}_{e^{\prime}}$ satisfy (4.10). At this point, (4.9) (4.14) are satisfied. If $e^{\prime} \neq e^{\prime \prime}$, then we have $\tilde{z}_{Q}\left(e^{\prime}\right)=\hat{x}_{Q}\left(e^{\prime}\right)=\hat{x}_{P}\left(e^{\prime}\right)$, which implies (4.15) for $e^{\prime}$. In order to ensure (4.15) for $e^{\prime \prime}$, we replace $\tilde{z}_{Q}\left(e^{\prime \prime}\right)$ by $+\infty$ if $e^{\prime \prime} \in U$. Since we have $\hat{x}_{Q}\left(e^{\prime \prime}\right)<\hat{x}_{P}\left(e^{\prime \prime}\right)$, this modification does not destroy (4.10), by Lemma 4.5. Hence, in (Case 1 ), the modified vectors satisfy all the required conditions.

Next we consider (Case 2), where $\hat{x}_{Q}(e)=\hat{x}_{P}(e)$ for all $e \in L$. In this case, we modify $\tilde{p}$ as well as $\hat{x}_{P}, \hat{x}_{Q}$, and $\tilde{z}_{Q}$ (while we keep $\tilde{z}_{P}$ the same). The procedure given below is based on a successive shortest path algorithm for finding a maximizer of the sum of two M-concave functions (Moriguchi and Murota [17], also see Iwata et al. [14]). It may be recognized as a sophisticated procedure for decreasing prices in auction algorithms. We deal with two functions defined by

$$
\begin{aligned}
& \hat{f}_{\bar{P}}^{\leq}\left(x_{0}, x\right)=\left\{\begin{array}{cl}
f_{P}(x) & \text { if } x_{0}=-x(E),\left(x_{0}, x\right) \leq \tilde{z}_{P} \\
-\infty & \text { otherwise }
\end{array} \quad\left(\forall\left(x_{0}, x\right) \in \mathbf{Z}^{\tilde{E}}\right),\right. \\
& \hat{f}_{\bar{Q}}^{\leq}\left(x_{0}, x\right)=\left\{\begin{array}{cl}
f_{Q}(x) & \text { if } x_{0}=-x(E),\left(x_{0}, x\right) \leq \tilde{z}_{Q} \\
-\infty & \text { otherwise }
\end{array} \quad\left(\forall\left(x_{0}, x\right) \in \mathbf{Z}^{\tilde{E}}\right) .\right.
\end{aligned}
$$

Obviously, $\hat{f}_{\bar{P}}^{\leq}$and $\hat{f}_{\bar{Q}}^{\leq}$are also M-concave, and $\hat{x}_{P} \in \arg \max \hat{f}_{\bar{P}}^{\leq}[+\tilde{p}]$ and $\hat{x}_{Q} \in$ $\arg \max \hat{f}_{\bar{Q}}^{\leq}[-\tilde{p}]$ hold.

We construct a directed graph $G=(\tilde{E}, A)$ and an arc length function $\ell: A \rightarrow$ $\mathbf{R} \cup\{+\infty\}$ as follows. Arc set $A$ consists of two disjoint parts:

$$
\begin{aligned}
& A_{P}=\left\{\left(e, e^{\prime}\right) \mid e, e^{\prime} \in \tilde{E}, e \neq e^{\prime}, \hat{x}_{P}-\tilde{\chi}_{e}+\tilde{\chi}_{e^{\prime}} \in \operatorname{dom} \hat{f}_{\bar{P}}^{\leq}\right\}, \\
& A_{Q}=\left\{\left(e, e^{\prime}\right) \mid e, e^{\prime} \in \tilde{E}, e \neq e^{\prime}, \hat{x}_{Q}+\tilde{\chi}_{e}-\tilde{\chi}_{e^{\prime}} \in \operatorname{dom} \hat{f}_{\bar{Q}}^{\leq}\right\},
\end{aligned}
$$

and $\ell \in \mathbf{R}^{A}$ is defined by

$$
\ell(a)= \begin{cases}\hat{f}_{\bar{P}}^{\leq}[+\tilde{p}]\left(\hat{x}_{P}\right)-\hat{f}_{\bar{P}}^{\leq}[+\tilde{p}]\left(\hat{x}_{P}-\tilde{\chi}_{e}+\tilde{\chi}_{e^{\prime}}\right) & \text { if } a=\left(e, e^{\prime}\right) \in A_{P} \\ \hat{f}_{Q}^{\leq}[-\tilde{p}]\left(\hat{x}_{Q}\right)-\hat{f}_{\bar{Q}}^{\leq}[-\tilde{p}]\left(\hat{x}_{Q}+\tilde{\chi}_{e}-\tilde{\chi}_{e^{\prime}}\right) & \text { if } a=\left(e, e^{\prime}\right) \in A_{Q} .\end{cases}
$$

Length function $\ell$ is nonnegative due to Theorem 4.1.

Let $S=\operatorname{supp}^{+}\left(\hat{x}_{P}-\hat{x}_{Q}\right)$ and $T=\{0\} \cup L \cup U$. We note that $S \cap T=\emptyset$ because $0 \notin S$ by $(4.14)$ and $\hat{x}_{Q} \neq \hat{x}_{P}$ and because $\hat{x}_{Q}(e)=\hat{x}_{P}(e)$ for all $e \in L \cup U$ by (4.15) and the assumption in (Case 2). Let $d: \tilde{E} \rightarrow \mathbf{R} \cup\{+\infty\}$ denote the shortest distances from $S$ to all vertices in $G$ with respect to $\ell$. For any $a=\left(e, e^{\prime}\right) \in A$ with $d(e)<+\infty$ we have

$$
\ell(a)+d(e)-d\left(e^{\prime}\right) \geq 0
$$


We note that there always exists a path from $S$ to $T$ because $(e, 0) \in A_{P}$ for all $e \in S$ by $\left(\mathrm{A}^{\prime}\right)$. Let $\mathbf{P}$ be a shortest path from $S$ to $T$ with the minimum number of arcs. We define $\delta$ by

$$
\delta=\min \{\ell(\mathbf{P}), \min \{\tilde{p}(e)-\underline{\pi}(e)+d(e) \mid e \in \tilde{E}\}\},
$$

where $\ell(\mathbf{P})=\sum_{a \in \mathbf{P}} \ell(a)$, and define a vector $\Delta \tilde{p}$ by

$$
\Delta \tilde{p}(e)=\min \{d(e)-\delta, 0\} \quad(\forall e \in \tilde{E}) .
$$

Obviously $\Delta \tilde{p} \leq \mathbf{0}$ holds. It follows from (4.20) (4.22) and the nonnegativity of $\ell$ that

$$
\ell(a)+\Delta \tilde{p}(e)-\Delta \tilde{p}\left(e^{\prime}\right) \geq 0 \quad\left(\forall a=\left(e, e^{\prime}\right) \in A\right) .
$$

The above system of inequalities is equivalent to

$$
\begin{aligned}
& \hat{f}_{\bar{P}}^{\leq}[+\tilde{p}]\left(\hat{x}_{P}\right)-\hat{f}_{\bar{P}}^{\leq}[+\tilde{p}]\left(\hat{x}_{P}-\tilde{\chi}_{e}+\tilde{\chi}_{e^{\prime}}\right)+\Delta \tilde{p}(e)-\Delta \tilde{p}\left(e^{\prime}\right) \geq 0 \\
& \hat{f}_{\bar{Q}}^{\leq}[-\tilde{p}]\left(\hat{x}_{Q}\right)-\hat{f}_{\bar{Q}}^{\leq}[-\tilde{p}]\left(\hat{x}_{Q}+\tilde{\chi}_{e}-\tilde{\chi}_{e^{\prime}}\right)+\Delta \tilde{p}(e)-\Delta \tilde{p}\left(e^{\prime}\right) \geq 0
\end{aligned}
$$

which is further equivalent to

$$
\hat{x}_{P} \in \arg \max \hat{f}_{\bar{P}}^{\leq}[+(\tilde{p}+\Delta \tilde{p})], \quad \hat{x}_{Q} \in \arg \max \hat{f}_{\bar{Q}}^{\leq}[-(\tilde{p}+\Delta \tilde{p})],
$$

due to Theorem 4.1. We show that $\tilde{p}+\Delta \tilde{p}$ satisfies (4.11). Since $\Delta \tilde{p} \leq \mathbf{0}$, it is enough to show that $\underline{\pi}(e) \leq \tilde{p}(e)+\Delta \tilde{p}(e)$ for all $e \in E$ and $\Delta \tilde{p}(0)=0$. Since $\delta \leq \ell(\mathbf{P})$, we have

$$
\Delta \tilde{p}(e)=0 \quad(\forall e \in T) .
$$

It follows from (4.21) that for all $e \in \tilde{E}$ we have

$$
\begin{aligned}
\tilde{p}(e)+\Delta \tilde{p}(e) & =\min \{\tilde{p}(e)+d(e)-\delta, \tilde{p}(e)\} \\
& \geq \min \{\tilde{p}(e)+d(e)-(\tilde{p}(e)+d(e)-\underline{\pi}(e)), \tilde{p}(e)\} \\
& =\underline{\pi}(e) .
\end{aligned}
$$

Thus, $\hat{x}_{P}, \hat{x}_{Q}, \tilde{p}+\Delta \tilde{p}, \tilde{z}_{P}$, and $\tilde{z}_{Q}$ satisfy Conditions $(4.9) \sim(4.15)$.

The above calculation shows that if $\delta<\ell(\mathbf{P})$, then there exists $e \in E$ with $\tilde{p}(e)>\tilde{p}(e)+\Delta \tilde{p}(e)=\underline{\pi}(e)$, that is, $L$ is enlarged. We next deal with the case where $\delta=\ell(\mathbf{P})$.

Suppose $\delta=\ell(\mathbf{P})$. Note that for each arc $a=\left(e, e^{\prime}\right) \in A, \ell^{\prime}(a)=\ell(a)+$ $\Delta \tilde{p}(e)-\Delta \tilde{p}\left(e^{\prime}\right)$ is the length of $a$ in the directed graph defined in the same way as above for $\hat{f}_{\bar{P}}^{\leq}[+(\tilde{p}+\Delta \tilde{p})], \hat{f}_{\bar{Q}}^{\leq}[-(\tilde{p}+\Delta \tilde{p})], \hat{x}_{P}$, and $\hat{x}_{Q}$. Since $\delta=\ell(\mathbf{P})$, we have $\ell^{\prime}(a)=0$ for all arcs $a \in \mathbf{P}$. Therefore, we have

$$
\begin{array}{ll}
\hat{x}_{P}-\tilde{\chi}_{e}+\tilde{\chi}_{e^{\prime}} \in \arg \max \hat{f}_{\bar{P}}^{\leq}[+(\tilde{p}+\Delta \tilde{p})] & \left(\forall\left(e, e^{\prime}\right) \in \mathbf{P} \cap A_{P}\right), \\
\hat{x}_{Q}+\tilde{\chi}_{e}-\tilde{\chi}_{e^{\prime}} \in \arg \max \hat{f}_{\bar{Q}}^{\leq}[-(\tilde{p}+\Delta \tilde{p})] & \left(\forall\left(e, e^{\prime}\right) \in \mathbf{P} \cap A_{Q}\right) .
\end{array}
$$


Since $\mathbf{P}$ has the minimum number of arcs, we have

$$
\begin{aligned}
& \hat{x}_{P}-\tilde{\chi}_{e}+\tilde{\chi}_{e^{\prime \prime}} \notin \arg \max \hat{f}_{\bar{P}}^{\leq}[+(\tilde{p}+\Delta \tilde{p})], \\
& \hat{x}_{Q}+\tilde{\chi}_{e}-\tilde{\chi}_{e^{\prime \prime}} \notin \arg \max \hat{f}_{\bar{Q}}^{\leq}[-(\tilde{p}+\Delta \tilde{p})]
\end{aligned}
$$

for all vertices $e$ and $e^{\prime \prime}$ of $\mathbf{P}$ such that $\left(e, e^{\prime \prime}\right) \notin \mathbf{P}$ and $e$ appears earlier than $e^{\prime \prime}$ in $\mathbf{P}$. Furthermore, arcs of $A_{P}$ and $A_{Q}$ appear alternately in $\mathbf{P}$. For otherwise, assume that two consecutive arcs $\left(e, e^{\prime}\right),\left(e^{\prime}, e^{\prime \prime}\right) \in \mathbf{P}$ belong to $A_{P}$ and then, by (M) we have

$$
\begin{aligned}
& \hat{f}_{\bar{P}}^{\leq}[+\tilde{p}]\left(\hat{x}_{P}-\tilde{\chi}_{e}+\tilde{\chi}_{e^{\prime}}\right)+\hat{f}_{\bar{P}}^{\leq}[+\tilde{p}]\left(\hat{x}_{P}-\tilde{\chi}_{e^{\prime}}+\tilde{\chi}_{e^{\prime \prime}}\right) \\
\leq & \hat{f}_{\bar{P}}^{\leq}[+\tilde{p}]\left(\hat{x}_{P}\right)+\hat{f}_{\bar{P}}^{\leq}[+\tilde{p}]\left(\hat{x}_{P}-\tilde{\chi}_{e}+\tilde{\chi}_{e^{\prime \prime}}\right),
\end{aligned}
$$

which yields

$$
\ell\left(e, e^{\prime}\right)+\ell\left(e^{\prime}, e^{\prime \prime}\right) \geq \ell\left(e, e^{\prime \prime}\right),
$$

a contradiction to the minimality of the number of arcs in $\mathbf{P}$. Consequently, we have

$$
\begin{aligned}
& a_{1}=\left(e_{1}, e_{1}^{\prime}\right), a_{2}=\left(e_{2}, e_{2}^{\prime}\right) \in \mathbf{P} \cap A_{P}, a_{1} \neq a_{2} \quad \Longrightarrow \quad\left\{e_{1}, e_{1}^{\prime}\right\} \cap\left\{e_{2}, e_{2}^{\prime}\right\}=\emptyset, \\
& a_{1}=\left(e_{1}, e_{1}^{\prime}\right), a_{2}=\left(e_{2}, e_{2}^{\prime}\right) \in \mathbf{P} \cap A_{Q}, a_{1} \neq a_{2} \Longrightarrow\left\{e_{1}, e_{1}^{\prime}\right\} \cap\left\{e_{2}, e_{2}^{\prime}\right\}=\emptyset .
\end{aligned}
$$

From Lemmas 4.2 and 4.3 together with (4.24) (4.26), we have

$$
\begin{aligned}
& \hat{x}_{P}^{\prime}=\hat{x}_{P}-\sum_{\left(e, e^{\prime}\right) \in \mathbf{P} \cap A_{P}}\left(\tilde{\chi}_{e}-\tilde{\chi}_{e^{\prime}}\right) \in \arg \max \hat{f}_{\bar{P}}^{\leq}[+(\tilde{p}+\Delta \tilde{p})], \\
& \hat{x}_{Q}^{\prime}=\hat{x}_{Q}+\sum_{\left(e, e^{\prime}\right) \in \mathbf{P} \cap A_{Q}}\left(\tilde{\chi}_{e}-\tilde{\chi}_{e^{\prime}}\right) \in \arg \max \hat{f}_{\bar{Q}}^{\leq}[-(\tilde{p}+\Delta \tilde{p})] .
\end{aligned}
$$

We replace $\hat{x}_{P}, \hat{x}_{Q}$ and $\tilde{p}$ by $\hat{x}_{P}^{\prime}, \hat{x}_{Q}^{\prime}$ and $\tilde{p}+\Delta \tilde{p}$, respectively. Modifications (4.27) and (4.28) guarantee that (4.9), (4.10) and (4.14) hold for modified vectors. We have already shown that $(4.11)$ holds. Since $\tilde{z}_{P}$ and $\tilde{z}_{Q}$ remain the same, Condition (4.23) implies (4.12) and (4.13). Let $e^{\prime}$ be the terminal vertex of $\mathbf{P}$ and let $a^{*}$ be the last arc of $\mathbf{P}$. If $e^{\prime} \notin U$, then (4.15) trivially holds. Hence we assume that $e^{\prime} \in U$ in the sequel.

If $a^{*} \in A_{Q}$, then we have $\hat{x}_{Q}\left(e^{\prime}\right)<\tilde{z}_{Q}\left(e^{\prime}\right)=\hat{x}_{P}\left(e^{\prime}\right)$ and (4.10). Hence, it follows from Lemma 4.5 that we can put $\tilde{z}_{Q}\left(e^{\prime}\right):=+\infty$ while preserving (4.10) and (4.15).

Suppose that $a^{*}$ belongs to $A_{P}$. Thus, $\hat{x}_{P}\left(e^{\prime}\right)=\hat{x}_{Q}\left(e^{\prime}\right)+1=\tilde{z}_{Q}\left(e^{\prime}\right)+1$ holds at this point. We increase $\tilde{z}_{Q}\left(e^{\prime}\right)$ by one. By (b) of Lemma 4.4 , there exists $e^{\prime \prime} \in \tilde{E}$ such that $\hat{x}_{Q}:=\hat{x}_{Q}+\tilde{\chi}_{e^{\prime}}-\tilde{\chi}_{e^{\prime \prime}}$ satisfy (4.10) for the updated $\tilde{z}_{Q}$. If $e^{\prime \prime} \in U$, then we put $\tilde{z}_{Q}\left(e^{\prime \prime}\right):=+\infty$. In the same way as in the argument for (Case 1 ), this modification yields (4.15) while preserving the other conditions.

Summarizing the above argument, we describe an algorithm PAIRWISE_STABLE. We will show that it terminates in a finite number of iterations and finds a pairwise strictly stable outcome. 


\section{Algorithm PAIRWISE_STABle}

Step 0. Find $\hat{x}_{P}, \hat{x}_{Q}, \tilde{p}, \tilde{z}_{P}$, and $\tilde{z}_{Q}$ satisfying (4.9) (4.15).

Step 1. If $\hat{x}_{P}=\hat{x}_{Q}$ then stop.

Step 2. Set $L$ and $U$ as (4.16) and (4.17). If there exists $e \in L$ with $\hat{x}_{Q}(e)<\hat{x}_{P}(e)$ then go to Step 3.a; else go to Step 4.a.

Step 3.a. Set $\tilde{z}_{P}(e):=\hat{x}_{P}(e)-1$ and $\hat{x}_{P}:=\hat{x}_{P}-\tilde{\chi}_{e}+\tilde{\chi}_{e^{\prime}}$, where $e^{\prime}$ is an element such that (4.9) is satisfied by the updated $\hat{x}_{P}$ and $\tilde{z}_{P}$.

3.b. If $e^{\prime} \notin U$ then go to Step 1; else go to Step 5 .

Step 4.a. Construct $G$ and compute $\ell$ for $\hat{f}_{\bar{P}}^{\leq}[+\tilde{p}], \hat{f}_{\bar{Q}}^{\leq}[-\tilde{p}], \hat{x}_{P}$ and $\hat{x}_{Q}$ by (4.18) and (4.19). Set $S:=\operatorname{supp}^{+}\left(\hat{x}_{P}-\hat{x}_{Q}\right)$ and $T:=\{0\} \cup L \cup U$. Compute the shortest distances $d(e)$ from $S$ to all $e \in \tilde{E}$ in $G$ with respect to $\ell$. Find a shortest path $\mathbf{P}$ from $S$ to $T$ with the minimum number of arcs.

4.b. Compute $\delta$ by (4.21). For each $e \in \tilde{E}$, set $\tilde{p}(e):=\tilde{p}(e)+\min \{d(e)-\delta, 0\}$. If $\delta<\ell(\mathbf{P})$ then go to Step 1.

4.c. Update $\hat{x}_{P}$ and $\hat{x}_{Q}$ by (4.27) and (4.28). If the terminal vertex $e^{\prime}$ of $\mathbf{P}$ is not in $U$ then go to Step 1.

4.d. If the last arc of $\mathbf{P}$ is in $A_{Q}$ then put $\tilde{z}_{Q}\left(e^{\prime}\right):=+\infty$ and go to Step 1 ; else go to Step 5.

Step 5. Set $\tilde{z}_{Q}:=\tilde{z}_{Q}+\tilde{\chi}_{e^{\prime}}$ and $\hat{x}_{Q}:=\hat{x}_{Q}+\tilde{\chi}_{e^{\prime}}-\tilde{\chi}_{e^{\prime \prime}}$, where $e^{\prime \prime}$ is an element such that (4.10) holds. If $e^{\prime \prime} \in U$ then set $\tilde{z}_{Q}\left(e^{\prime \prime}\right):=+\infty$. Go to Step 1.

We have already shown the following lemma.

Lemma 4.6: Conditions (4.9) (4.15) are satisfied at Step 1 in each iteration of PAIRWISE_STABLE.

The following two lemmas show the termination of PAIRWISE_STABLE.

Lemma 4.7: In each iteration of PAIRWISE_STABLE, the following statements hold.

(a) L enlarges or remains the same.

(b) $\tilde{z}_{P}$ decreases or remains the same.

(c) $\tilde{z}_{Q}$ increases or remains the same. 
(d) $\sum_{e \in E}\left(\hat{x}_{P}(e)-\hat{x}_{Q}(e)\right)=\hat{x}_{Q}(0)-\hat{x}_{P}(0)$ decreases or remains the same.

Proof. Since $L=\{e \in E \mid \tilde{p}(e)=\underline{\pi}(e)\}$ and $\tilde{p}$ does not increase during PAIRWise_Stable, we have (a). Statements (b) and (c) trivially hold. Furthermore, we obviously have $\sum_{e \in E}\left(\hat{x}_{P}(e)-\hat{x}_{Q}(e)\right)=\hat{x}_{Q}(0)-\hat{x}_{P}(0)$, which is nonnegative by (4.14). Vectors $\hat{x}_{P}$ and/or $\hat{x}_{Q}$ are modified at Step 3.a, Step 4.c or Step 5. At Step 3.a or Step 4.c, if $e^{\prime}=0$, then $\hat{x}_{Q}(0)-\hat{x}_{P}(0)$ is decreased by one; otherwise it remains the same. At Step 5 , if $e^{\prime \prime}=0$, then $\hat{x}_{Q}(0)-\hat{x}_{P}(0)$ is decreased by one; otherwise it remains the same. Hence (d) holds.

We denote by [StepXX $\rightarrow$ Step1] the case where we go from Step XX to Step 1 after execution of Step XX in PAIRWise_Stable.

Lemma 4.8: PAIRWISE_STABLE has the following features.

(a) In $\left[\right.$ Step3.b $\rightarrow$ Step1], some component of $\tilde{z}_{P}$ strictly decreases.

(b) In $[$ Step4.b $\rightarrow$ Step1], L strictly enlarges.

(c) In $\left[\right.$ Step4.c $\rightarrow$ Step1], either $\hat{x}_{Q}(0)-\hat{x}_{P}(0)$ strictly decreases or (a) occurs in the next iteration.

(d) In [Step4.d $\rightarrow$ Step1], some component of $\tilde{z}_{Q}$ strictly increases.

(e) In $\left[\right.$ Step $5 \rightarrow$ Step1], some component of $\tilde{z}_{Q}$ strictly increases.

Proof. (a): At the beginning of Step 3.a, we have $\tilde{z}_{P}(e) \geq \hat{x}_{P}(e)$. Hence, $\tilde{z}_{P}(e)$ strictly decreases at Step 3.a.

(b): As we have already shown, $L$ strictly enlarges when $\delta<\ell(\mathbf{P})$.

(c): In this case, we have either $e^{\prime}=0$ or $e^{\prime} \in L \backslash U$. In the former case $\hat{x}_{Q}(0)-\hat{x}_{P}(0)$ is decreased by one. Since $\hat{x}_{Q}(e)=\hat{x}_{P}(e)$ for all $e \in L \cup U$ at the beginning of Step 4, we have $\hat{x}_{P}\left(e^{\prime}\right)>\hat{x}_{Q}\left(e^{\prime}\right)$ for $e^{\prime}(\in L)$ at the end of Step 4.c, which results in (a) in the next iteration.

(d): In this case, we have $e^{\prime} \in U$ at the beginning of Step 4.d. Hence, $\tilde{z}_{Q}\left(e^{\prime}\right)$ strictly increases.

(e): If $e^{\prime} \neq e^{\prime \prime}$, then $\tilde{z}_{Q}\left(e^{\prime}\right)$ increases by one; otherwise $\tilde{z}_{Q}\left(e^{\prime}\right)=+\infty$.

Lemma 4.9: PAIRWISE_STABLE terminates in a finite number of iterations if $f_{P}$ and $f_{Q}$ satisfy $\left(\mathrm{A}^{\prime}\right)$.

Proof. Since $f_{P}$ and $f_{Q}$ satisfy $\left(\mathrm{A}^{\prime}\right)$, we have

- $\hat{x}_{Q}(0)-\hat{x}_{P}(0)$ is nonnegative and bounded from above, 
- if $\tilde{z}_{P}(e)<+\infty$ then it is nonnegative and bounded from above,

- if $\tilde{z}_{Q}(e)<+\infty$ then it is bounded from above by (4.15).

Hence, Lemmas 4.7 and 4.8 guarantee the termination of PAIRWISE_STABLE in finitely many steps.

Lemma 4.10: If $f_{P}$ and $f_{Q}$ are integer-valued on their effective domains, $\underline{\pi} \in$ $(\mathbf{Z} \cup\{-\infty\})^{E}$, and $\bar{\pi} \in(\mathbf{Z} \cup\{+\infty\})^{E}$, then $\tilde{p}$ is preserved to be integer-valued in PAIRWISE_STABLE.

Proof. Because $\bar{\pi} \in(\mathbf{Z} \cup\{+\infty\})^{E}, \tilde{p}$ is initially defined to be integer-valued in PAIRWISE_STABLE. Since $f_{P}$ and $f_{Q}$ are integer-valued on their effective domains, $\ell$ defined in (4.19) is integer-valued. Furthermore, as $\underline{\pi} \in(\mathbf{Z} \cup\{-\infty\})^{E}, \delta$ defined in (4.21) is also integer-valued. Hence, $\tilde{p}(e):=\tilde{p}(e)+\min \{d(e)-\delta, 0\}$ modified at Step 4.b is preserved to be integer for each $e \in \tilde{E}$.

By Lemmas 4.6 and 4.9, PAIRwise_Stable always finds $\hat{x}_{P}, \hat{x}_{Q}, \tilde{p}, \tilde{z}_{P}$, and $\tilde{z}_{Q}$ satisfying $(4.8) \sim(4.13)$ under $\left(\mathrm{A}^{\prime}\right)$. By Lemma 4.10 , if $f_{P}$ and $f_{Q}$ are integervalued on their effective domains, $\underline{\pi} \in(\mathbf{Z} \cup\{-\infty\})^{E}$, and $\bar{\pi} \in(\mathbf{Z} \cup\{+\infty\})^{E}$, then $p$ is preserved to be integer-valued. This completes the proof of Theorem 3.5.

\section{Acknowledgements}

This work is supported by a Grant-in-Aid for Scientific Research from the Ministry of Education, Culture, Sports, Science and Technology of Japan.

\section{References}

[1] A. Alkan and D. Gale, Stable schedule matching under revealed preference, J. Econom. Theory 112 (2003), 289-306.

[2] V. Danilov, G. Koshevoy, and C. Lang, Gross substitution, discrete convexity, and submodularity, Discrete Appl. Math. 131 (2003), 283-298.

[3] A. Eguchi, S. Fujishige, and A. Tamura, A generalized Gale-Shapley algorithm for a discrete-concave stable-marriage model, Algorithms and Computation: 14th International Symposium, ISAAC2003, LNCS 2906 (T. Ibaraki, N. Katoh, and H. Ono, eds.), Springer-Verlag, Berlin, 2003, pp. 495-504.

[4] K. Eriksson, and J. Karlander, Stable matching in a common generalization of the marriage and assignment models, Discrete Math. 217 (2000), 135-156. 
[5] R. Farooq and A. Shioura, A note on the equivalence between substitutability and $M^{\natural}$-convexity, Pacific J. Optim. to appear.

[6] R. Farooq and A. Tamura, A new characterization of $M^{\natural}$-convex set functions by substitutability, J. Oper. Res. Soc. Japan 47 (2004), 18-24.

[7] T. Fleiner, A matroid generalization of the stable matching polytope, Integer Programming and Combinatorial Optimization: 8th International IPCO Conference, LNCS 2081 (B. Gerards and K. Aardal, eds.), Springer-Verlag, Berlin, 2001, pp. 105-114.

[8] T. Fleiner, A fixed point approach to stable matchings and some applications, Math. Oper. Res. 28 (2003), 103-126.

[9] S. Fujishige, Submodular Functions and Optimization, Annals of Discrete Mathematics 47, North-Holland, Amsterdam, 1991.

[10] S. Fujishige and A. Tamura, A general two-sided matching market with discrete concave utility functions, RIMS Preprint 1401, Kyoto University, 2003, http://www.kurims.kyoto-u.ac.jp/home_page/preprint/list/.

[11] S. Fujishige and Z. Yang, A note on Kelso and Crawford's gross substitutes condition, Math. Oper. Res. 28 (2003), 463-469.

[12] D. Gale and L. S. Shapley, College admissions and the stability of marriage, Amer. Math. Monthly 69 (1962), 9-15.

[13] F. Gul and F. Stacchetti, Walrasian equilibrium with gross substitutes, J. Econom. Theory 87 (1999), 95-124.

[14] S. Iwata, S. Moriguchi, and K. Murota, Capacity scaling algorithm for Mconvex submodular flow, Integer Programming and Combinatorial Optimization: 10th International IPCO Conference, LNCS 3064 (D. Bienstoc and G. Nemhauser, eds.), Springer-Verlag, Berlin, 2004, pp. 352-367.

[15] M. Kaneko, The central assignment game and the assignment markets, J. Math. Econom. 10 (1982), 205-232.

[16] J. A. S. Kelso and V. P. Crawford, Job matching, coalition formation, and gross substitutes, Econometrica 50 (1982), 1483-1504.

[17] S. Moriguchi and K. Murota, Capacity scaling algorithm for scalable M-convex submodular flow problems, Optim. Methods Softw. 18 (2003), 207-218.

[18] K. Murota, Convexity and Steinitz's exchange property, Adv. Math. 124 (1996), 272-311. 
[19] K. Murota, Discrete convex analysis, Math. Programming 83 (1998), 313-371.

[20] K. Murota, Discrete Convex Analysis, Society for Industrial and Applied Mathematics, Philadelphia, 2003.

[21] K. Murota and A. Shioura, M-convex function on generalized polymatroid, Math. Oper. Res. 24 (1999), 95-105.

[22] K. Murota and A. Shioura Relationship of $M$ - /L-convex functions with discrete convex functions by Miller and by Favati-Tardella, Discrete Appl. Math. 115 (2001), 151-176.

[23] K. Murota and A. Tamura, New characterizations of M-convex functions and their applications to economic equilibrium models with indivisibilities, Discrete Appl. Math. 131 (2003), 495-512.

[24] A. E. Roth and M. A. O. Sotomayor, Two-Sided Matching - A Study in Game-Theoretic Modeling and Analysis, Cambridge University Press, Cambridge, 1990.

[25] A. E. Roth and M. A. O. Sotomayor, Stable outcomes in discrete and continuous models of two-sided matching: A unified treatment, Rev. Econom. 16 (1996), 1-24.

[26] L. S. Shapley and M. Shubik, The assignment game I: The core, Internat. J. Game Theory 1 (1972), 111-130.

[27] M. Sotomayor, Existence of stable outcomes and the lattice property for a unified matching market, Math. Social Sci. 39 (2000), 119-132. 